\title{
Primary and secondary thrombocytosis induced by exercise and environmental luminosity
}

\author{
Beck WR ${ }^{1,2}$, Scariot PPM², Gobatto $\mathrm{CA}^{2}$ \\ Physical Education Faculty, University of Campinas, Campinas, Sao Paulo, Brazil. cgobatto@uol.com.br
}

\begin{abstract}
Objectives: We investigated the exercise and different environmental luminosities effects on blood platelets count in order to identify primary and secondary thrombocytosis, respectively.

Background: Platelets alteration has been associated with important pathological events, such as neurodegenerative diseases, and the count of these cells in bloodstream is influenced by several effects, including physical and chemical. Owing the difficulty to study the aetiology of thrombocytosis in human models, we employed acute and chronic free drug interventions in order to identify these two types of this important disease in laboratory animals. Methods: Forty rats were exposed to standard (SI) or experimental (EI) illumination from 45 days-old. Both groups were exposed to $12 \mathrm{~h}$ daylight $(2700 \mathrm{~K} ; 565-590 \mathrm{~nm} ;<60 \mathrm{lux}$; from 06:00 $\mathrm{h}$ to 18:00 h). During dark period SI animals were kept in total darkness while El remained under red light (> $600 \mathrm{~nm},<15$ lux). At 92 days-old, exercised animals were submitted to an acute bout of swimming at individualized intensity and control animals remained at rest.

Results: Blood samples were collected immediately after the exercise for platelets count, which were among $849000 \pm 115817$ and $1085600 \pm 177089 / \mathrm{mm}^{3}$ of blood. Exercise $(F=6.91 ; p=0.01)$ and $\mathrm{El}(F=6.66 ; p=$ $0.01)$ increased platelets count, showing no interaction between effects $(F=0.01 ; p=0.89)$.

Conclusion: Primary thrombocytosis was detected owing an acute exercise and the secondary thrombocytosis due to the constant red light during dark period, without any pharmacological interventions and strongly respecting the ethical aspects, enabling future studies on aetiology of thrombocytosis through this model (Fig. 2, Ref. 35). Text in PDF www.elis.sk.

Key words: thrombocytosis, platelets, exercise, animal model, environmental luminosity.
\end{abstract}

Megakaryocytopoiesis is the production of megakaryocytes, responsible for platelets release (1). Platelets are important discshaped small anuclear cells of blood that play an important role repairing wounds and the vascular damage naturally generating during all life, beyond the participation on innate immune response (2) and clotting process (3) widely investigated currently due to the important source of information for biological science (4-6). Platelets alteration has been associated with some important pathological events, such as neurodegenerative diseases (7). The blood platelets count in blood stream is influenced by several effects, including physical and chemical (8). The primary thrombocytosis (increased platelets count) is acute-phase related, while secondary is usually associated to chronic events (9).

${ }^{1}$ Physical Education Faculty, University of Campinas, Campinas, Sao Paulo, Brazil, and ${ }^{2}$ Laboratory of Applied Sport Physiology, School of Applied Sciences, University of Campinas, Limeira, Sao Paulo, Brazil

Address for correspondence: C.A. Gobatto, Laboratory of Applied Sport Physiology, School of Applied Sciences, Department of Sport Sciences, University of Campinas. Pedro Zaccaria Street, 1.300, Jardim Santa Luíza - Postal Code 13484-350 - Limeira - Sao Paulo. Phone: +55.19.37016653, Fax: +55.19.37016680

Acknowledgements: This study was funded by the Sao Paulo State Research Support Foundation (FAPESP), Proc. 2010/13377-7 and 2011/13226-1.
It is quite known that the exercise transiently increases platelet count (10), which are initially released by the spleen and lungs (8) probably due to the muscular microtrauma (11). This primary thrombocytosis is characterized by short lived increase, considered as a normal organism response toward benefit exercise adaptation. Nevertheless, secondary thrombocytosis is considered abnormal and usually related to chronic inflammation, myocardial infarction, cancer, thrombosis and cardiovascular diseases $(1,8,12)$.

In clinical routine it is very difficult to determine if the thrombocytosis is related to primary or secondary mechanisms in human beings (9). Thus, the animal model provides a unique tool to better understand some diseases and healing process (1) and could be useful for aetiology and pathogenesis of thrombocytosis, however, this possibility should be more studied. The rat is the most used animal on experiments, however, this rodent are nocturnal (13) and possess a high light sensibility (14). This sensibility associated to variations on environmental luminosity could generate stress, which could lead to secondary thrombocytosis (15).

In light of the above findings, our study investigated the acute effect of a swimming bout at anaerobic threshold intensity until exhaustion and the chronic effect of different environmental illuminations on blood platelets count. Our hypothesis was that with this design we would be capable of distinguish the primary and secondary thrombocytosis. Accepting this, the 
$607-610$

primary and secondary thrombocytosis could be induced without any pharmacological interventions and strongly respecting the ethical aspects.

\section{Methods}

\section{Animals}

Forty albino Wistar rats were kept in polyethylene cages at 22 $\pm 2{ }^{\circ} \mathrm{C}, 45-55 \%$ of relative humidity and noise below 85 decibels, being a light/dark cycle of 12/12 h with lights switched on at 06:00 h. The animals were fed with rat-specific standard chow (70 \% carbohydrate, $23.5 \%$ protein, $6.5 \%$ fat; Labina-Purina ${ }^{\circledR}$, Purina 5008, St. Louis, MO) and water ad libitum. The institutional review board approved the experiment (protocol 018/10), which followed the current National Legislation and Standards for Animal Experimentation.

\section{Environmental luminosity and study design}

During the light period all animals were exposed to an incandescent lamp (Phillips ${ }^{\circledR}$, Soft, 100 W, 2700 K, 565-590 nm, < 60 lux). At 45 days old, forty rats were equally divided into 2 groups: standard (SI) and experimental illumination (EI). From 18:00 h to 06:00 h, EI groups were exposed to constant red light (Rosco ${ }^{\circledR}$, Supergel, Fire \# 19, > 600 nm, < 15 lux), while SI groups remained at total darkness and were submitted to the same red light only during procedures. Lamps were carefully positioned to assure equal distance among light source and cages.

Handling, cage cleaning, water and food addition and testing were begun at 20:00h in order to respect the high values for body core temperature (16) and spontaneous activity of rats (17), leading the animals to less stress at this time of day (18). In order to perform two weeks of an aquatic and swimming testing adaptation (AA) the animals were subdivided into control (ct) and exercised (ex) groups at 76 days-old. At the end of AA and at 90 days-old, the lactate minimum test (LMT) were conducted for all animals. After 48 hours, exercised groups were submitted to a swimming bout until exhaustion at lactate minimum intensity (tlim), while control groups remained at rest (Fig. 1).

\section{Swimming testing}

Routines were performed in cylindrical PVC tubes $(30 \mathrm{~cm}$ diameter and $120 \mathrm{~cm}$ depth) containing clean water at $31 \pm 1{ }^{\circ} \mathrm{C}$, to elicit individual swimming and warrant the continuous swimming pattern.

The lactate minimum test (LMT) consisted in three steps: 1 ) hyperlactatemia induction by two short bouts of high intensity exercise (13\% of body weight - $\%$ bw); 2 ) passive recovery period of 9 minutes to allow the release of the lactate produced by muscles toward the bloodstream; and 3) incremental phase (4, 4.5, 5, 5.5, $6,7 \%$ bw), in which the blood lactate was collected and analyzed for the minimum value identification. This nadir was calculated through second order polynomial adjust and indicated the maximal intensity when the release and consumption of blood lactate was equal, representing the maximal lactate steady state phenomena in swimming rats (19). The lactate minimum intensity (LMi) and

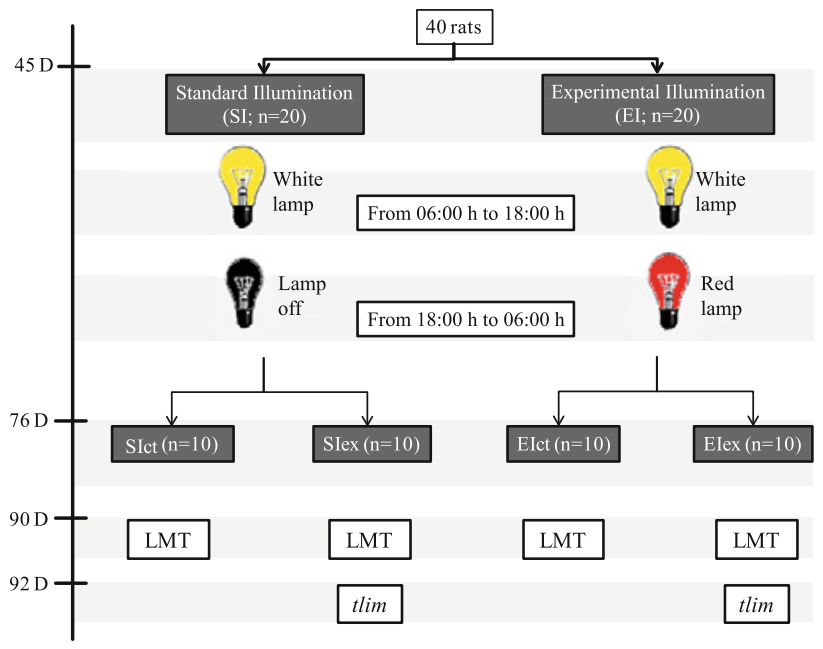

Fig. 1. Schematic summary of study design. SIct - standard illumination control; SIex - standard illumination exercised; EI - experimental illumination; LMT - lactate minimum test; tlim - time to exhaustion.

lactatemia corresponding to LMi (LLM) were determined as described elsewhere (20).

Forty-eight hours after perform LMT, exercised animals were submitted to a swimming trial at lactate minimum intensity, being recorded the time to exhaustion (tlim). The exhaustion criteria was accept unanimously by two experienced researchers, when the animal was incapable to return to the surface during 10 seconds submerged, even with vigorous trying, as previously described (21).

\section{Blood collection and analytical procedures}

Blood samples were collected from rat's distal tail during LMT, before ([lac]pre) and after ([lac]post) tlim. After prepared, samples were used to determine lactate concentration by enzymatic method against calibration curve, as described elsewhere (22). Immediately after tlim for the exercised group, or at rest and same time of day for control group, animals were euthanized in carbonic gas chamber, being blood collected by cardiac puncture method. Blood samples of each rat was immediately transferred to a plastic tubes containing k3EDTA (fl Medical ${ }^{\circledR}$, Torreglia, PD, Itália) for platelets counts, which were analyzed by hemocromocytometric flux method with semiconductor laser performed on automatic counter (MAXM Coulter).

\section{Statistical procedures}

Data were expressed as the mean \pm standard deviation. Statistical analysis was carried out employing a software package (Statistic 7.0). We used the t test to determine the differences between the exercised groups for LMi, LLM, [lac]pre and [lac]post and tlim. Two-way analysis of variance were used for the investigation of luminosity and exercise effects (and their interaction) on platelets count. The Newmann-Keuls post hoc test was used to locate differences when pertinent. The significance level was set at $5 \%(\mathrm{p}<0.05)$. 


\section{Results}

LMi corresponded to $4.78 \pm 0.27$ and $4.98 \pm 0.27 \%$ bw for SI and EI ( $p=0.12$ ), respectively, being the lactatemia for these intensities corresponding to $5.81 \pm 1.00$ and $4.38 \pm 0.52 \mathrm{mmol} / \mathrm{L}$ ( $<<0.01)$, respectively.

The tlim corresponded to $113.58 \pm 46.67$ for SI and $51.93 \pm$ $24.65 \mathrm{~min}$ for $\mathrm{EI}(\mathrm{p}<0.01)$. [lac]pre corresponded to $1.99 \pm 0.42$ $\mathrm{mmol} / \mathrm{L}$ for SI and $1.21 \pm 0.25 \mathrm{mmol} / \mathrm{L}$ for EI $(\mathrm{p}<0.01)$ and [lac] post were $5.78 \pm 0.87$ and $5.63 \pm 1.21 \mathrm{mmol} / \mathrm{L}$ for SI and EI, respectively $(\mathrm{p}=0.76)$.

Platelets count for SIct, SIex, EIct e EIex were $849000 \pm$ 115817, $974400 \pm 146827,972200 \pm 114645$ and $1085600 \pm$ $177089 / \mathrm{mm}^{3}$ of blood. The exercise significantly increased platelets counts $(\mathrm{F}=6.91 ; \mathrm{p}=0.01)$ as well as the experimental illumination $(F=6.66 ; \mathrm{p}=0.01)$, with no interaction between these main effects $(F=0.01 ; p=0.89)$, as illustrated by Figure 2 .

\section{Discussion}

The main finding of the present study was the identification of primary and secondary thrombocytosis through acute and chronic effects owing to exercise and environmental luminosity, respectively, accepting our initial hypothesis.

Concerning the lactate minimum test, we found no luminosity effect for LMi, revealing the protocol's robustness. Moreover, the LLM and [lac]pre were sensible to identify the adverse condition, at which the animals were exposed when under EI, considering that in some previous studies on malnourished rats the same lactatemia results were found (23). Probably the adverse conditions increased adrenocorticotropic hormone (24) and consequently adrenaline and corticosterone (25). This situation led to an increase in resting glycolytic metabolism, decreasing glycogen content (26).

Exercise and luminosity effects

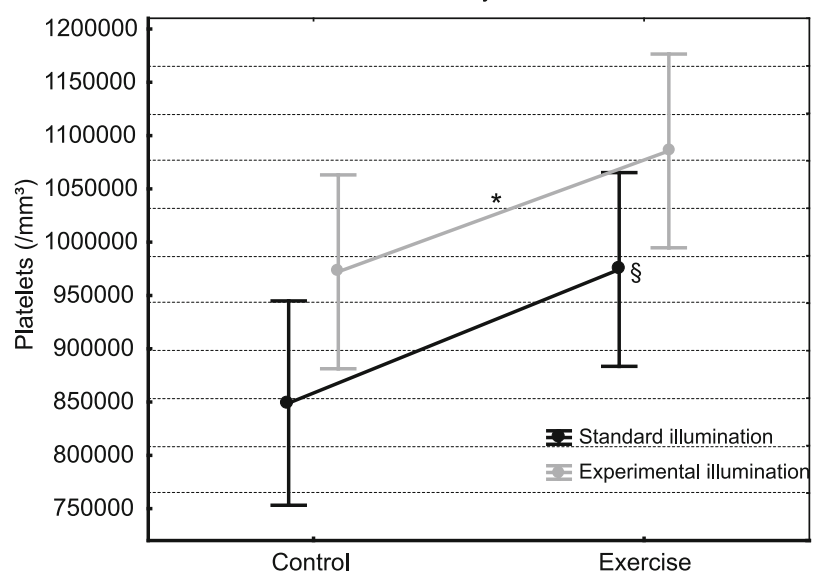

Fig. 2. ANOVA results for exercise (control vs exercise), luminosity (standard vs experimental illumination) and their interaction for platelets count. $* \mathrm{~F}=6.66$ and $\mathrm{p}=0.01$ in relation to $\mathrm{SI} ; \S \mathrm{F}=6.91$ and $\mathrm{p}=$ 0.01 in relation to control. The effects showed no significant interaction as described in the text.
Considering that less glycogen is available, glycolytic substrate is reduced and also the lactate concentration $(23,27)$. According to these authors and in agreement of this logic, low lactate concentration could indicate low glycogen stores. Since the glycogen is the main substrate for the required intensity (28) the lower tlim for EI is a reasonable result.

Regarding the exercise effect on platelets count, even with no statistical difference among control and exercise groups for SI and EI, we found an increment of $12.87 \%$ and $10.45 \%$, respectively, which was enough to lead to a significant effect of exercise when analyzed by analysis of variance $(F=6.91 ; p=0.01)$. Animal model experiments showed that despite the platelets decreased due to exercise training in Wistar rats (29), an acute exercise increased these counts (30). Moreover, there was a great variation on platelets activation regarding to endurance exercise in literature (31), being the activation associated to the factors explained in the introduction section, as muscular microtrauma releasing platelets from the spleen and lungs $(8,11)$. Considering these known platelets variation owing to a specific exercise intensity, we individually prescribed the exercise through lactate minimum test, a robust and valid protocol contained in swimming rats literature (19, 20, 32).

In addition, higher platelets counts were found for animals exposed to experimental illumination (secondary thrombocytosis), in other words, chronically. This finding could be explained due to the possible stress originated by constant red light during dark period. Stressful conditions has been related to modulations in platelet count and some disorders related to platelet functionality in humans $(15,33,34)$. Regarding the above described, our study showed an evidence in the same direction for rats.

We could observe in the results section that both exercise and luminosity generated significant increase on platelets count. Moreover, there was no interaction between effects, which demonstrated that the exercise or experimental luminosity could modulate the platelets count singly. According to our interpretation, this result confirms the presence of acute and chronic effects on platelets count owing exercise and luminosity, respectively.

Despite the fact that our study discriminated the primary and secondary thrombocytosis using a rodent model, our design is not out of criticism. The platelets activation and aggregation certainly could conduct us to more robust affirmations, however, platelet scores could be related to platelets activation and aggregation (35). For instance, with the platelets count we could investigate our initial hypothesis, nevertheless, future studies are necessary to deeply explore the mechanisms of platelets activation and aggregation for primary and secondary thrombocytosis generated by the studied effects.

In summary, we found higher platelets counts in animals exercised and submitted to an experimental illumination when compared to control and standard illumination. The primary thrombocytosis was found due to an acute strenuous exercise and the secondary thrombocytosis was found owing the stress caused by the constant red light during dark period for these nocturnal animals. We accepted the initial hypothesis, determining a useful rodent model to induce primary and secondary thrombocytosis without drugs administration and respecting the ethical aspects for animal experimentations, allowing future studies supported 
$607-610$

by molecular analysis the view to enhancing the knowledge about this important disease.

\section{References}

1. Carmichael CL, Alexander WS. Mouse models of diseases of megakaryocyte and platelet homeostasis. Mammalian Genome 2011; 22 (7-8): 449-465.

2. Kaushansky K. The molecular mechanisms that control thrombopoiesis. J Clin Invest 2005; 115 (12): 3339-3347.

3. Ahmadizad S, El-Sayed MS, Maclaren DP. Responses of platelet activation and function to a single bout of resistance exercise and recovery. Clin Hemorheol Microcirculat 2006; 35 (1-2): 159-168.

4. Nayak MK, Kulkarni PP, Dash D. Regulatory role of proteasome in determination of platelet life span. J Biol Chem 2013.

5. Cimmino G, Golino P. Platelet Biology and Receptor Pathways. J Cardiovasc Translat Res 2013.

6. Behari M, Shrivastava M. Role of platelets in neurodegenerative diseases: a universal pathophysiology. Internat J Neurosci 2013: Epub ahead of print.

7. Shrivastava M, Vivekanandhan S. An insight into ultrastructural and morphological alterations of platelets in neurodegenerative diseases. Ultrastruc Pathol 2011; 35 (3): 110-116.

8. El-Sayed MS, Ali N, El-Sayed Ali Z. Aggregation and activation of blood platelets in exercise and training. Sports Med 2005; 35 (1): 11-22.

9. Griesshammer M, Bangerter M, Sauer T, Wennauer R, Bergmann L, Heimpel H. Aetiology and clinical significance of thrombocytosis: analysis of 732 patients with an elevated platelet count. J Intern Med 1999; 245 (3): 295-300.

10. El-Sayed MS. Effects of exercise on blood coagulation, fibrinolysis and platelet aggregation. Sports Med 1996; 22 (5): 282-298.

11. Fehrenbach E, Schneider ME. Trauma-induced systemic inflammatory response versus exercise-induced immunomodulatory effects. Sports Med 2006; 36 (5): 373-384.

12. Kaushansky K. Determinants of platelet number and regulation of thrombopoiesis. Hematology / the Education Program of the American Society of Hematology American Society of Hematology Education Program 2009: 147-152.

13. Könczöl K, Pinter O, Ferenczi S, Varga J, Kovacs K, Palkovits M et al. Nesfatin-1 exerts long-term effect on food intake and body temperature. Internat J Obesity 2012; 36 (12): 1514-1521.

14. Albarracin R, Eells J, Valter K. Photobiomodulation protects the retina from light-induced photoreceptor degeneration. Invest Ophthalmol Visual Sci 2011; 52 (6): 3582-3592.

15. Brydon L, Magid K, Steptoe A. Platelets, coronary heart disease, and stress. Brain Behav Immunity 2006; 20 (2): 113-119.

16. Tanaka H, Yanase M, Kanosue K, Nakayama T. Circadian variation of thermoregulatory responses during exercise in rats. Amer J Physiol 1990; 258 (4 Pt 2): R836-841.

17. Ikeda M, Sagara M, Inoue S. Continuous exposure to dim illumination uncouples temporal patterns of sleep, body temperature, locomotion and drinking behavior in the rat. Neurosci Lett 2000; 279 (3): 185-189.

18. Pittendrigh CS, Daan SA. A functional analysis of circadian pacemakers in nocturnal rodents. IV. Entrainment: pacemaker as clock. J Comp Physiol 1976; 106: 291-331.
19. de Araujo GG, Papoti M, Manchado Fde B, de Mello MA, Gobatto CA. Protocols for hyperlactatemia induction in the lactate minimum test adapted to swimming rats. Comparative biochemistry and physiology Part A. Mol Integrat Physiol 2007; 148 (4): 888-892.

20. Beck WR, de Araujo GG, Gobatto CA. Methods of exercise intensity and lactataemia determination of lactate minimum test in rats. Compar Exercise Physiol 2012; 8 (2): 113-116.

21. McArdle WD, Montoye HJ. Reliability of exhaustive swimming in the laboratory rat. J Appl Physiol 1966; 21 (4): 1431-1434.

22. Engel PC, Jones JB. Causes and elimination of erratic blanks in enzymatic metabolite assays involving the use of NAD+ in alkaline hydrazine buffers: improved conditions for the assay of L-glutamate, L-lactate, and other metabolites. Analyt Biochem 1978; 88 (2): 475-484.

23. Voltarelli FA, Gobatto CA, de Mello MAR. Determinaçao da transiçao metabólica através do teste do lactato mínimo em ratos desnutridos durante exercício de nataçao. R da Educaçao Física/UEM 2007; 18 (1): 33-39.

24. Aguilera G. Regulation of pituitary ACTH secretion during chronic stress. Front Neuroendocrinol 1994; 15: 321-350.

25. Axelrod J, Reisine TD. Stress hormones: their interaction and regulation. Science 1984; 224 (4648): 452-459.

26. Kolataj A, Rysinska J, Flak P. The influence of selection on reaction to stress in mice II. Influence of ACTH, adrenalin and insulin on the aldolase and LDH activity in liver and kidney. J Anim Breed Genet 1992; 109: 144-148.

27. Voltarelli FA, de Mello MAR, Gobatto CA. Glicogenio muscular e limiar anaeróbio determinado em ratos durante a natacao. Motriz 2004; 10 (1): 25-30.

28. Beneke R, Hutler M, Leithauser RM. Maximal lactate-steady-state independent of performance. Med Sci Sports Exercise 2000; 32 (6): 11351139.

29. Wang JS, Lin CC, Chen JK, Wong MK. Role of chronic exercise in decreasing oxidized LDL-potentiated platelet activation by enhancing platelet-derived no release and bioactivity in rats. Life Sci 2000; 66 (20): 1937-1948.

30. Ficicilar H, Zergeroglu AM, Tekin D, Ersoz G. The effects of acute exercise on plasma antioxidant status and platelet response. Thrombosis Res 2003; 111 (4-5): 267-271.

31. El-Sayed MS, El-Sayed Ali Z, Ahmadizad S. Exercise and training effects on blood haemostasis in health and disease: an update. Sports Med2004; 34 (3): 181-200.

32. de Araujo GG, Papoti M, Dos Reis IG, de Mello MA, Gobatto CA. Physiological responses during linear periodized training in rats. Eur J Appl Physiol. 2012; 112 (3): 839-852.

33. Baltrusch HJ, Andres J, Stangel W. Psychosocial stress, personality and blood platelet behavior: implications for psychophysiologic and psychoneuroimmunologic research. Internat J Neurosci 1990; 51 (3-4): 237-239.

34. Steptoe A, Magid K, Edwards S, Brydon L, Hong Y, Erusalimsky J. The influence of psychological stress and socioeconomic status on platelet activation in men. Atherosclerosis 2003; 168 (1): 57-63.

35. Lee KW, Lip GY. Acute versus habitual exercise, thrombogenesis and exercise intensity. Thromb Haemostas 2004; 91 (3): 416-419.

Received April 1, 2013. Accepted May 15, 2014. 